\title{
Some New Methods of Time Series Analysis: Applications to the AGB Stars
}

\section{I.L. Andronov}

Department of Astronomy, Odessa State University

\begin{abstract}
Some supplementary methods of time series analysis are described which are used for study of periodic and aperiodic variability of the AGB stars. These are "multiharmonic fits" used to fit the periodic (asinusoidal) curve; running approximations - "parabolae", "sines"; "asymptotic parabolae"; "linear fits".

Stellar variability exhibits a variety of combinations of the contributions of variations of different time scale and coherence. We analyze the light curves in two main aspects: a) the determination of the main parameters of the mean phase light curve of the stars of some type and further study of correlations of them with other physical characteristics and b) study of cycle-to-cycle changes of individual stars and search for slow variations and the search for possible correlations between various parameters.

As a general tool for approximations the method of least squares is used. However, different types of variability suggest necessity of diversity of the basic functions and weights used for the fit. For stable period(s) we use a set of programs using one- or multi- frequency, multi-shift or multi-harmonic fits as described by Andronov (1994). To determine parameters of the individual cycles we use running approximations, mainly the "running parabolae fit" (Andronov, 1990, 1996). Using this method, we determine maxima and minima. For the additional points of crossing the constant brightness value the local linear fit is preferred.

In the case of large data gaps more effective may be the "running sine" fit (Chinarova et al 1994) which may gather data point from few nearby cycles. It may be extended to "few harmonic" running fit which is better for the asymmetric curves. The number of harmonics is to be determined by using the Fischer's test. Such fit is good to look for changes with timescale larger than the interval of smoothing. However, the cycle-to-cycle changes may be masked.

The light curves of many Mira-type stars may be well described as practically linear ascending and descending branches connected by relatively short nearly parabolic branches at maximum or minimum. In this case it is natural to choose basic functions with asymptotes instead of usual or trigonometric polynomials. This method of "asymptotic parabolae" is very effective, despite its more complex algorithm described by Marsakova and Andronov (1996), especially for data with large gaps and extrema shifted to a limit of the observational interval.
\end{abstract}

\section{REFERENCES}

Andronov, I.L., 1990, Kinemat. Fizika Nebesn. Tel, 6, N 6, 87.

Andronov, I.L., 1994, Odessa Astron. Publ., 7, 49.

Andronov, I.L., 1996, Astron. Astrophys., subm.

Chinarova, L.L., Andronov, I.L., Schweitzer, E., 1994, Odessa Astron. Publ., 7, 103.

Marsakova, V.I., Andronov, I.L., 1996, Odessa Astron. Publ., 9, (in press). 